\title{
The diagnostic process of Kyrle's disease in a 65-year-old patient
}

\author{
Szymon Leonik ${ }^{1}$, Michał Smoczok ${ }^{1}$, Katarzyna Kulig ${ }^{2}$, Beata Bergler-Czop ${ }^{3}$, Bartosz Miziołek ${ }^{4}$
}

${ }^{1}$ Student Scientific Society at the Department and Clinic of Dermatology, Medical University of Silesia, Katowice, Poland ${ }^{2}$ Chair and Department of Dermatology, Andrzej Mielęcki Silesian Independent Public Hospital, Katowice, Poland ${ }^{3}$ Department and Clinic of Dermatology, Medical University of Silesia, Katowice, Poland

${ }^{4}$ Department of Internal Medicine Rheumatology and Clinical Immunology, School of Medicine, Medical University of Silesia, Katowice, Poland

Adv Dermatol Allergol 2022; XXXIX (3): 620-622

DOI: https://doi.org/10.5114/ada.2021.108565

A 65-year-old patient was admitted to the ward for the diagnosis and treatment of hyperkeratotic papules, located around the follicles and forming plaques, as well as erosions covered with crusts up to $1.5 \mathrm{~cm}$ in diameter. The lesions were located in the dorsal part of the hands, forearms, with single foci on the lower legs (Figures $1 \mathrm{~A}, \mathrm{~B}$ ). The patient reported itching within the lesions. The patient's medical history is about 1 year long.

The patient had cardiologic and internal organ diseases. The medical history included the following: hypertension, ischaemic heart disease, paroxysmal atrial fibrillation, secondary diabetes (glycaemia $10.84 \mathrm{mmol} / \mathrm{l}$ ), secondary hyperparathyroidism (PTH - 549 pg/ml; hypocalcaemia $2.06 \mathrm{mmol} / \mathrm{l}$, hyperphosphataemia 2.49 $\mathrm{mmol} / \mathrm{l}$, and hyperkalaemia $5.61 \mathrm{mmol} / \mathrm{l})$, chronic kidney disease (creatinine $939.1 \mu \mathrm{mol} / \mathrm{l})$, chronic hepatitis B without delta virus, and previous acute pancreatitis. Furthermore, the patient had a cardiac pacemaker implanted due to third-degree atrioventricular block and coronary artery bypass grafting (CABG). The patient has been under insulin therapy for many years and haemodialysed for 7 years.

The differential diagnosis included the following: reactive acquired perforating collagenosis, perforating folliculitis, elastosis perforans serpiginosa, and the perforating form of granuloma. During the diagnostics, the patient was tested for antinuclear antibodies using indirect immunofluorescence, obtaining a homogeneous positive result with a titre of $1: 320$, and the same method was used to determine if any antibodies were directed against his own neutrophils (ANCA) - no antibodies were detected in the $1: 20$ ANCA titre. The blood serum was tested for antibodies for pemphigus/pemphigoid - nega- tive results for IgA and IgG with a $1: 20$ titre. In addition, the total IgE was negative.

During further diagnosis of skin lesions, a videodermatoscopic examination of the efflorescence revealed a zonal, concentric pattern, including the following: a central crust, a hyperkeratotic sheath, an unstructured pink area, and a few blood vessels (Figures 1 C, D). A skin specimen was collected for histopathological evaluation, which revealed the following: acanthosis and hyperkeratosis in the epidermis, especially around the follicles, and hyperplasia with inflammatory granulation deep into the skin. On the edge of the specimen, the epidermis was covered with a crust that may indicate ulceration or exit of the follicular fistula penetrating deep into the skin with signs of epithelial hyperplasia (Figures $1 \mathrm{E}, \mathrm{F}$ ). There were no clear diagnostic features, but the whole may correlate with Kyrle's disease. Taking into account the nature of the skin lesions, the medical history, the results of the laboratory tests, as well as the results of additional tests, Kyrle's disease was diagnosed.

Kyrle's disease (lat. hyperkeratosis follicularis et follicularis in cutempenetrans) was first diagnosed at the beginning of the $20^{\text {th }}$ century. It belongs to the relatively rarely described (its exact epidemiology is unknown) acquired dermatoses from the group of perforating dermatoses (this group also includes reactive perforating collagenosis, perforating folliculitis, and elastosis perforans serpiginosa) [1]. To this day, its aetiology remains unclear, although one of the leading theories is the occurrence of the keratinisation process in the basal layer of the epidermis which elicits an inflammatory response in the host [2]. It is characterised by a disorder of the keratinisation process within the epidermis leading to transepidermal elimination dis-

Address for correspondence: Szymon Leonik, Student Scientific Society at the Department and Clinic of Dermatology, Medical University of Silesia, 20-24 Francuska St, 40-027 Katowice, Poland, e-mail: leonikszymon@gmail.com Received: 27.07.2020, accepted: 3.11.2020. 

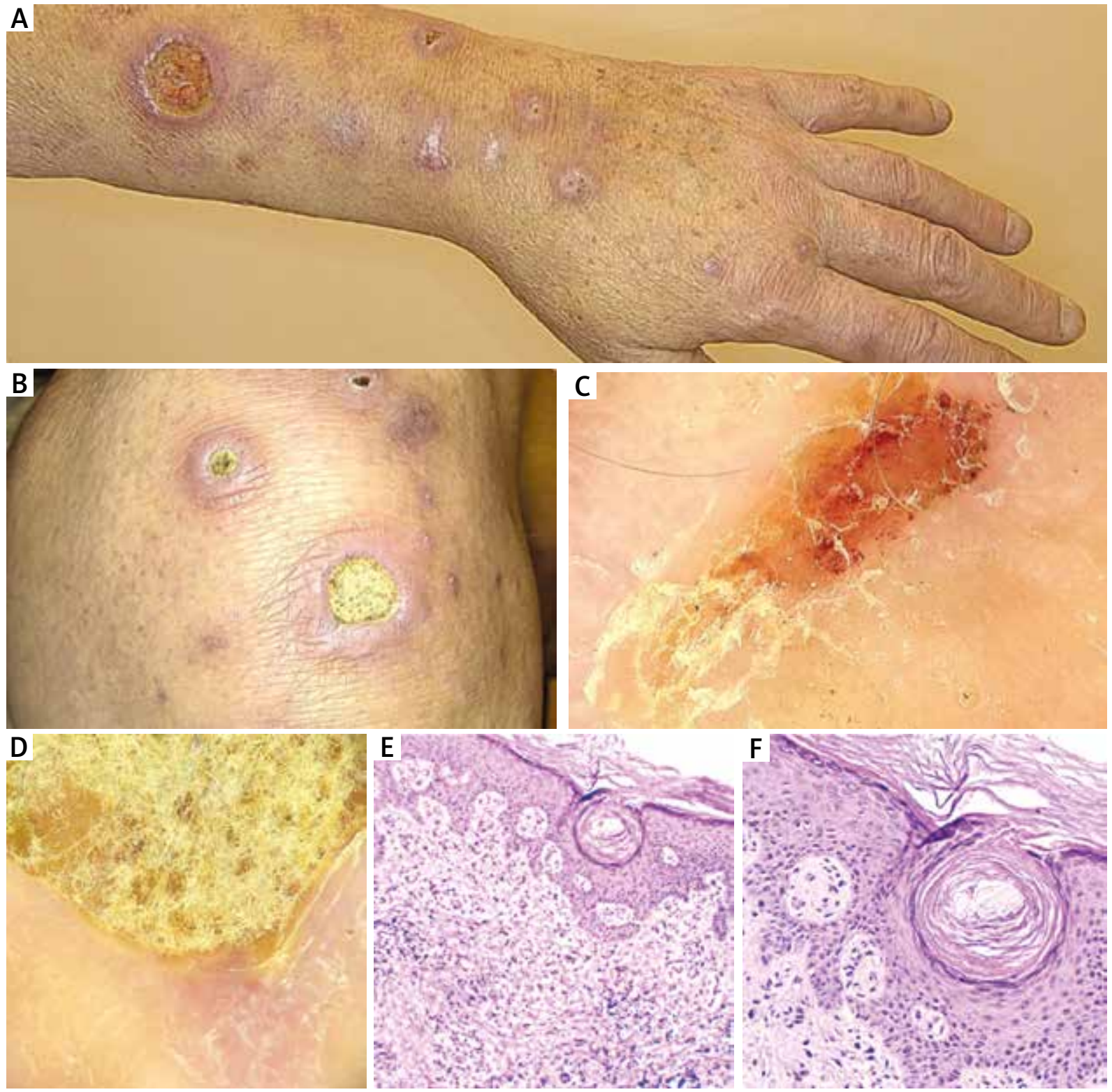

Figure 1. Patient's skin changes in the course of Kyrle's disease. Papules around the follicles clustering in plaques on the distal part of the upper limb, some lesions covered with a yellow crust. A, B - macroscopic images. C, D - video dermatoscopic images. E, F- microscopic images of the lesion (source - own study)

orders. The correlation between the occurrence of Kyrle's disease and metabolic disorders of patients seems to be significant. Cases of isolated disease onset have been reported less frequently. Diabetes is the main disorder correlating with Kyrle's disease. However, it also occurs in patients with impaired renal function (it is estimated that about $10 \%$ of patients on dialysis develop the disease), or liver or congenital heart disease [3, 4].

In its course, hyperkeratotic papules and plaques occur on the skin of patients [2]. Vesicular lesions may also occur within the lesions. Their location is variable. However, there is a tendency for these to occur in the distal parts of the body - especially in the lower limbs.
Kyrle's disease is a chronic disorder with a tendency for periodic remission and exacerbation. The data indicate the hereditary nature of some of the cases described; however, the majority of patients do not have a history of genetic predisposition. In the typical picture of the disease, we observe changes in the nature of the follicular keratosis and maculopapular eruptions, usually located on the distal parts of the limbs [5].

During system therapy, ceftriaxone $2 \mathrm{~g}$ IV/day was used. The topical treatment was clobetasol propionate in the morning and mupirocin in the evening. There was a gradual improvement of the condition. The patient was discharged after 12 days of hospitalization. It was recom- 
mended that the patient continue topical therapy treatment as an outpatient.

Retinoids (oral and topical preparations), topical glucocorticosteroids, light therapy (SUP311 and PUVA), as well as laser therapy $\left(\mathrm{CO}_{2}\right.$ laser for individual skin lesions), cryotherapy, and surgical treatment are also used in the treatment of Kyrle's disease [2].

\section{Conflict of interest}

The authors declare no conflict of interest.

\section{References}

1. Bodman M, Ehredt D Jr, Barker R, et al. Kyrle disease a rare dermatologic condition associated with the diabetic foot. J Am Podiatr Med Assoc 2015; 105: 451-5.

2. Płużańska-Srebrzyńska K, Gerlicz-Kowalczuk Z, Wojciechowska A, et al. Choroba Kyrlego. Forum Derm 2016; 2: 131-3.

3. Forouzandeh M, Stratman S, Yosipovitch G. The treatment of Kyrle's disease: a systematic review. J Eur Acad Dermatol Venereol 2020. doi: 10.1111/jdv.16182.; 34: 1457-63.

4. Nair PA, Jivani NB, Diwan NG. Kyrle's disease in a patient of diabetes mellitus and chronic renal failure on dialysis. J Family Med Prim Care 2015; 4: 284-6.

5. Russo T, Piccolo V, Mascolo M, et al. Dermoscopy of Kyrle disease. J Am Acad Dermatol 2016; 75: e99-101. 